\title{
XLVI. On the resultant of a number of unit vibrations, whose phases are at random over a range not limited to an integral number of periods
}

\section{Lord Rayleigh O.M. F.R.S.}

To cite this article: Lord Rayleigh O.M. F.R.S. (1919) XLVI. On the resultant of a number of unit vibrations, whose phases are at random over a range not limited to an integral number of periods, Philosophical Magazine Series 6, 37:221, 498-515, DOI: $10.1080 / 14786440508635910$

To link to this article: http://dx.doi.org/10.1080/14786440508635910

曲 Published online: 08 Apr 2009.

Submit your article to this journal

Џll Article views: 9

Q View related articles $\llbracket$

47 Citing articles: 6 View citing articles 지 
XLVI. On the Resultant of a Number of Unit Vibrations, whose Phases are at Random over a Range not limited to an Integral Number of Periods. By Lord RAYLEIGH, O.M., F.R.S.*

AUMBER $(n)$ of points is distributed at randon on a $A$ straight line of length $a$. When $n$ is very great, the centre of gravity of the points tends to coincidence with the middle point of the line, which is taken as origin of coordinates. What is the probability that the error of position, that is its deviation from the origin, lies between $x$ and $x+d x$ ?

Divide the length $a$ into a large odd number $(2 s+1)$ of parts, each equal to $b$. The number of points to be expected on each $b$ is $n b / a$. This expectation would be fulfilled in the mean of a large number of independent trials, but in a single trial it is subject to error. If the actnal number be $n b / a+\xi$, the chance that $\xi$ lies between $\xi$ and $\xi+d \xi$ is by Bernoulli's theorem

$$
\frac{d \xi}{\sqrt{(2 \pi n b / a)}} e^{-a \xi^{2}: 2 n t}, \text {. . . . . . }
$$

in which it is assumed that while $b / a$ is very small, $n b / a$ is nevertheless very great $t$. In the language of the Theory of Errors, the modulus, proportional to "probable error," is $\sqrt{ }(2 n b / a)$.

The points which fall on any small part $b$ may be treated as acting at the middle of the part. For instance, those which fall on the part which includes the origin are supposed to act at the origin and so make no contribution to the sum of the moments; while on other parts the moment is proportional to the distance between the middle of the part and the origin. Thus if

$$
\xi_{-s}, \xi_{-8+1}, \xi_{-s+2}, \ldots \xi_{-1}, \xi_{0}, \xi_{1}, \ldots \xi_{s}
$$

be the values of the various $\xi$ 's, the coordinate $x$ of the centre of gravity is given by

$$
x=\frac{b\left(\xi_{1}-\xi_{-1}\right)+2 b\left(\xi_{2}-\xi_{-2}\right)+\ldots+s b\left(\xi_{s}-\xi_{-s}\right)}{n+\xi_{-s}+\xi_{-8+1}+\ldots+\xi_{s}} .
$$

If the whole number of the points be $n$ exactly, the sum of the $\xi$ 's in the denominator of (2) must vanish exactly;

* Communicated by the Author.

† Compare Phil. Mag. vol. xlvii. p. 246 (1899). 
On the Resultant of a Number of Lnit Vibrations.

but if we assume this beforehand, the various $\xi$ 's are not independent, as is required by the rules of the Theory of Errors. We may evade the difficulty by supposing the value of $\xi$ on any part to be the result of an independent distribution of $n$ points over the whole length. The total of the $\xi$ 's is then not necessarily zero, but if we select those cases in which $n$ is zero, or nearly enough zero, the original requirement is fulfilled. In point of fact no selection is required, inasmuch as the probable error of the sum of $\xi$ 's is $\sqrt{ }(2 s+1)$ times the probable error of each and therefore proportional to $\sqrt{ }(2 s+1) \cdot \sqrt{ }(2 n b / a)$, or $V^{\prime}(2 n)$, so that no error of which there is a tinite probability is comparable with $n$. We may accordingly take (2) in the simplified form

$$
x=\frac{b}{n}\left\{\xi_{1}-\xi_{-1}+2\left(\xi_{2}-\xi_{-2}\right)+\ldots+s\left(\xi_{s}-\xi_{-8}\right)\right\} ;
$$

and the (modulus) ${ }^{2}$ for the composite error $x$ is given by

$$
\frac{\operatorname{Mod}^{2} x}{\operatorname{Mod}^{2} \xi}=\frac{2 b^{2}}{n^{2}}\left(1^{2}+2^{2}+3^{2}+\ldots+s^{2}\right) .
$$

For our purpose the sum of the series may be identified with $\int_{0}^{s} s^{2} d s$, or $s^{3} / 3$, or if we prefer it, $(2 s+1)^{3} / 24$, that is $a^{3} / 24 b^{3}$, and thus

$$
\operatorname{Mod}^{2} \text { for } x=a^{2} / 6 n, \text {. . . . . }
$$

$s$, as well as $n$, being regarded as infinitely great.

'The probability of an error between $x$ and $x+d x$ in the position of the centre of gravity of the $n$ points is accordingly

$$
\sqrt{\left(\frac{6 n}{\pi}\right) e^{-6 n x^{2} / a^{2}} d x / a, \ldots . .} \text {. . }
$$

showing in what manner the probability of a finite $x$ becomes infinitely small as $n$ increases without limit.

The method bitherto employed requires that the total number $(n)$ of points be very great. It is of interest also to inquire what are the various probabilities when $n$ is small or moderate. In dealing with this problem it seems more convenient to reckon the distances from one end of the line $a$, and to calculate in the first instance the chances for the $\operatorname{sum}(\sigma)$ of the distances. We take $\phi_{n}(\sigma) d \sigma / a$ to represent the chance that for $n$ points this sum lies between $\sigma$ and $\sigma+d \sigma$, and we commence with a sequence formula connecting $\phi_{n+1}$ with $\phi_{n}$. If for the-moment we suppose $2 \mathrm{M} 2$ 
$\phi_{n}$ known and consider the inclusion of an additional point, we see that

$$
\phi_{n+1}(\sigma)=\int_{\sigma-a}^{\sigma} \phi_{n}(\sigma) d \sigma / a . \quad . \quad . \quad .
$$

By means of (6) the various functions may be built up in order.

We start from $\phi_{1}(\sigma)$. This is zero, unless $0<\sigma<a$, and then is unity. Hence between 0 and $a$

$$
\phi_{2}(\sigma)=\int_{0}^{\sigma} \phi_{1}(\sigma) d \sigma / a=\sigma / a .
$$

If $\sigma$ lies between $a$ and $2 a$,

Thus

$$
\phi_{2}(\sigma)=\int_{\sigma-\alpha}^{a} \phi_{1}(\sigma) d \sigma / a=\frac{2 a-\sigma}{a} .
$$

$$
\left.\begin{array}{l}
\phi_{2}(\sigma)=0, \quad(\sigma<0) ; \quad \phi_{2}(\sigma)=\sigma / a, \quad(0<\sigma<a) ; \\
\phi_{2}(\sigma)=(2 a-\sigma) / a ; \quad(a<\sigma<2 a) ; \quad \phi_{2}(\sigma)=0,(2 a<\sigma)
\end{array}\right\},
$$

by which $\phi_{2}$ is completely determined; and it will be seen that there is no breach of continuity in the values of $\phi_{2}$. itself at the critical places. These values are symmetrical on the two sides of $\sigma=a$, and can be represented on a diagram by two straight lines passing through $\sigma=0$ and $\sigma=2 \alpha$, and meeting at $\sigma=a$. (See fig. 1.)

In like manner we can deduce $\phi_{3}$ from $\phi_{2}$. If $\sigma<0$, $\phi_{3}=0$, and indeed generally $\phi_{n}=0$. If $0<\sigma<a$,

If $a<\sigma<2 a$,

$$
\phi_{3}(\sigma)=\int_{0}^{\sigma} \frac{\sigma d \sigma}{a^{2}}=\frac{\sigma^{2}}{2 a^{2}} .
$$

$$
\phi_{3}(\sigma)=\int_{\sigma-a}^{a} \frac{\sigma d \sigma}{a^{2}}+\int_{a}^{\sigma} \frac{2 a-\sigma}{a^{2}} d \sigma=\left(-3 a^{2}+3 a \sigma-\sigma^{2}\right) / a^{2}
$$

From the symmetry it follows that when $2 a<\sigma<3 a$,

$$
\phi_{3}(\sigma)=(3 a-\sigma)^{2} / 2 a^{2} \text {. }
$$

When

$$
\sigma>3 a, \quad \phi_{3}(\sigma)=0 .
$$

It may be remarked that in this case not only is $\phi_{3}$ continuous, but also the first derivative $\phi_{3}{ }^{\prime}$. The representative curves for all three portions are parabolic. The maximum of $\phi_{3}$, occurring at $\sigma=3 a / 2$, is $3 / 4$. 
These problems might also be attacked in another and perhaps more direct manner by expressing the probabilities as multiple definite integrals. Thus in the case of two points the chance of distances $x$ and $y$ from the chosen end is $d x d y / a^{2}$, and what we require is the integral of this taken between the proper limits. If we treat $x$ and $y$ as rectangular coordinates of a point lying within the square whose side is $a$, the probability we seek is represented by the length of the line within the square which is drawn perpendicular to the diagonal through the origin, $\sigma$ itself corresponding to the position of the line as measured along the diagonal.

For three points we have to consider a cube of side $a$, when the chance is represented in like manner by the area within the cube of a plane drawn perpendicularly to the diagonal through the origin. At first, that is near the origin, the area is triangular and increases as $\sigma^{2}$; afterwards it becomes hexagonal, and after passing through the form of a regular hexagon, when its area is a maximum, returns backwards through the same phases.

The calculations by the sequence formula present no difficulty of principle. When $n=4$, I find

$$
\begin{gathered}
(0<\sigma<a), \quad \phi_{4}(\sigma)=\sigma^{3} / \hat{\cup} a^{3} ; \\
(a<\sigma<2 a), \quad \phi_{4}(\sigma)=\left\{\sigma^{3}-4(\sigma-a)^{3}\right\} / 6 a^{3} ;
\end{gathered}
$$

when $2 a<s<4 a$, the above values are repeated symmetrically. In this case there is no discontinuity either in $\phi_{4}$, or $\phi_{4}{ }^{\prime}$, or $\phi_{4}{ }^{\prime \prime}$. When $\sigma=2 \alpha$, that is in the middle of the range,

$$
\phi_{4}=2 / 3, \quad \phi_{4}^{\prime}=0 .
$$

The calculations might be pursued to higher values of $n$ without much trouble. In all cases there is symmetry with respect to the middle of the range. The functions $\phi_{n}$ are algebraic and rise in degree by a unit at each step. At the beginning of the range $\phi_{n+1}(\sigma)=(\sigma / a)^{n} / n !$, so that the contact at both ends of the representative curves with the line of abscissæ becomes of high order.

Again, since $\sigma$ must lie somewhere between 0 and $n a$, we must have

$$
\int_{0}^{n a} \phi_{n}(\sigma) d \sigma / a=1 ; \ldots . . .
$$

from the above expressions we may test this in the cases of $n=2,3,4$. 
A plot of the curves for these cases is given in fig. 1. The ordinate represents $\phi(\sigma)$ and the abscissa represents $\sigma$ itself with $a$ taken as unity, so that the area of each curve is unity.

Fig. 1.
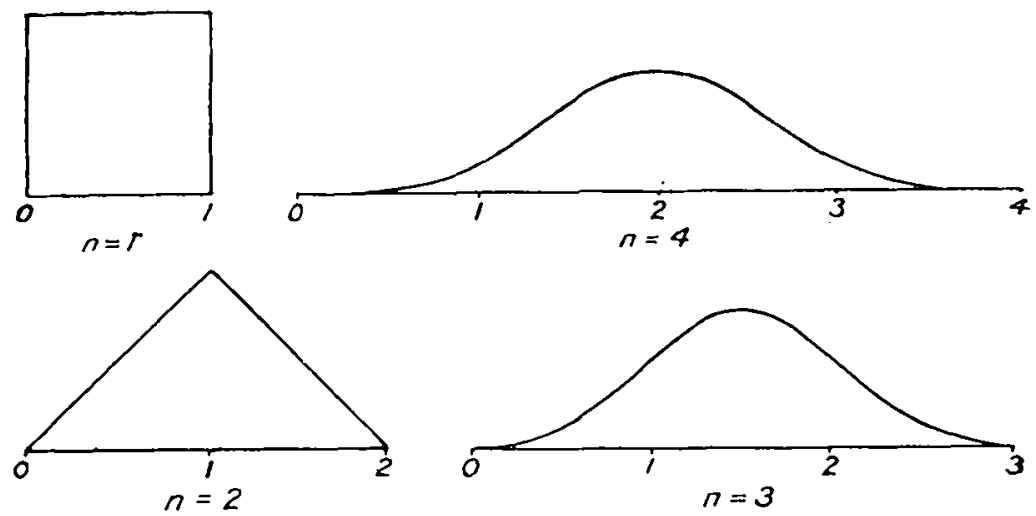

In order to pass from these curves in which $\sigma$ is the sum of the distances from one end to the represontative curves for the mean distance, which must, lie between 0 and $a$, wo have merely to reduce the scale of the absciss $x$ in the ratio $n: 1$, and to increase the scale of the ordinates in the same ratio, so that the area is preserved. For instance. when $n=4$, the middle ordinate will be increased from $2 / 3$ to $8 / 3$.

The sequence formula (6). serves well enough for the derivation of the facility curves appropriate to moderate values of $n$, but it does not lend itself readily to examination of the passage towards the final form when $n$ is great. This purnose is better attained by an adaptation of a remarkable method due to Laplace *, and employed by him and by Airy $t$ for the derivation of the usual exponential formula for the facility of error. Here again it will be the sum of the distances of the points, now reckoned from the middle of the line, that we consider in the first instance.

The distances, instead of being continuously distributed are supposed to be limited to definite values, all equally probable,

$$
-s l,(-s+1) b,(-s+2) b, \ldots .-l, 0, l, 2 b, \ldots . s l,
$$

* See 'lodhunter's 'History of the Thenry of l'robability' p. 521 .

+ 'Theory of Firor of Obeercution, Nacmillan, 1861, p. 8. In a comparison of the present notation with that of Laplace and Airy, the symbols $n$ and $s$ will be geen to be interchanged. 
where $2 s b=a$, and ultimately $s$ will be made infinite. The question is-What is the chance that the sum of the distances of $n$ points sball be equal to $l b$, where $l$ is a positive or negative integer? On eximnination it appears that the combination follows the same laws "as the addition of indices in the successive multiplication of the polynomial

$$
e^{-i s \theta}+e^{-i(s-1 ; \theta}+e^{-i(s-2) \theta}+\ldots+e^{2(s-2) \theta}+e^{i(s-1) \theta}+e^{i s \theta}
$$

by itself, supposing the operation repeated $n-1$ times. And therefore the number of combinations required will be the coefficient of $e^{i l \theta}$ (which is also the same as the coefficient of $\left.e^{-i l \theta}\right)$ in the expransion of

$$
\left\{e^{-i \theta \theta}+e^{-i(s-1) \theta}+\ldots+e^{i(\theta-1) \theta}+e^{i \lambda \theta}\right\}^{n} \text {. }
$$

The number of combinations required is therefore the same as the term independent of $\theta$ in the expansion of

$$
\frac{1}{2}\left(e^{i l \theta}+e^{-i l \theta}\right)\left\{e^{-i s \theta}+e^{-i(s-1) \theta}+\ldots+e^{i(s-1) \theta}+e^{i s \theta}\right\}^{n},
$$

or the same as the term independent of $\theta$," when

$$
\cos l \theta\{1+2 \cos \theta+2 \cos 2 \theta+\ldots+2 \cos s \theta\}^{n}
$$

is expanded and arranged according to cosines of multiples of $\theta$. By summing the series and application of Fourier's theorem this term is found to be

$$
\frac{1}{\pi} \int_{0}^{\pi} \cos l \theta\left\{\frac{\sin \frac{1}{2}(2 s+1) \theta}{\sin \frac{1}{2} \theta}\right\}^{n} d \theta . . .
$$

This is the number of combinations which gives rise to a sum equal to $l$, and in order to obtain the probability of $l$ it must be divided by the whole number of combinations equally probable, that is $(2 s+1)^{n}$. What we have to consider is accurdingly the value of

$$
\frac{1}{\pi(2 s+1)^{n}} \int_{0}^{\pi} \cos l \theta\left\{\frac{\sin \frac{1}{2}(2 s+1) \theta}{\sin \frac{1}{2} \theta}\right\}^{n} d \theta .
$$

In their discussion, Laplace and Airy regard both $n$ and $s$ as infinite. Here it is proposed to make $s$ infinite, so as to ittain a continuous distribution of the points, but without limitation upon the value of $n$, which may be any integer. If, as befoie, $\sigma$ denote the sum of the distances,

$$
\sigma=l b=l a \mid 2 s \text {. }
$$

When $s$ is very great, $\sin s \theta$ alternates with great rapidity, 
as soon as $\theta$ becomes sensible, so that the integral comes tis depend upon that part of the range where $\theta$ is very sinall. We may then replace $\sin \frac{1}{2} \theta$ by $\frac{1}{2} \theta$, and taking $\psi=\theta / s$, we find

$$
\frac{1}{s \pi} \int_{0}^{\infty} \cos \frac{2 \sigma \psi}{a} \frac{\sin ^{n} \psi}{\psi^{n}} d \psi \text {. . . . . }
$$

as the equivalent of $(10)$ when $s$ becomes infinite. This is the probability which attaches to a single integral value of $l$, or to a change $d \sigma$, where $d \sigma=a / 2 s$. Thus the probability that $\sigma$ lies between $\sigma$ and $\sigma+d \sigma$ may be written

$$
\frac{2 d \sigma}{a \pi} \int_{0}^{\infty} \cos \frac{2 \sigma \psi}{a} \frac{\sin ^{n} \psi}{\psi^{2}} d \psi
$$

which is the required result for a continuous distribution and is applicable to any value of $n$. In our former notation,

$$
\phi_{n}(\sigma)=\frac{2}{\pi} \int_{0}^{\infty} \cos \frac{2 \sigma \psi}{a} \frac{\sin ^{n} \psi}{\psi^{n}} d \psi
$$

in which, however, $\sigma$ now represents the sum of tire distances from the centre of the line, instead of from one end of it.

If $n=1$, (13) reduces to

$$
\phi_{1}(\sigma)=\frac{1}{\pi} \int_{0}^{\infty} \frac{\sin (1+2 \sigma / a) \psi+\sin (1-2 \sigma / a) \psi}{\psi} d \psi,
$$

which is unity when $\sigma$ lies between $\pm \frac{1}{2} a$, but otluerwise vanishes.

Again, if $n=2$, we find that $\phi_{2}{ }^{\prime}(\sigma)= \pm 1 / a$, if $\sigma$ lies between $\pm a$, and otherwise vanishes, and so on.

More generally, the sequence formula may be deduced from (11), but to obtain it in the original form (6), where the distances are measured from the end of the line, we must write $\sigma-\frac{1}{2} n a$ for $\sigma$ in (11). Then we have

$$
\frac{2}{\pi} \int_{0}^{\infty} \int_{\sigma-a}^{\sigma} \cos \frac{2 \psi}{a}\left(\sigma-\frac{1}{2} n a\right) \cdot \frac{\sin ^{n} \psi}{\psi^{n}} d \psi d \sigma / a,
$$

in which

$$
\int_{\sigma-a}^{\sigma} \cos \frac{2 \psi}{a}\left(\sigma-\frac{1}{2} n a\right) d \sigma=\cos \frac{2 \psi}{a}\left(\sigma-\frac{n+1}{2} a\right) \cdot \frac{\sin \psi}{\psi}
$$

so that (11) is verified.

We may now examine the form assumed by $\phi_{n}$ in (13), when $n$ is very large. The process is almost the same as 
that followed in a recent paper *. By taking logarithms we find

where

$$
\frac{\sin ^{n} \psi}{\psi^{n}}=e^{-n \psi^{2} / 6}\left\{1+n h_{4} \psi^{4}+n h_{6} \psi^{6}+\frac{1}{2} n^{2} h_{4}^{2} \psi^{8}\right\}
$$

$$
l_{4}=-\frac{1}{180}, \quad l_{6}=-\frac{1}{35 \cdot \overline{8} \cdot .} . \quad .
$$

Retaining for the moment only the leading term, we get

$$
\begin{aligned}
\phi_{n}(\sigma) d \sigma / a & =\frac{d \sigma}{a} \frac{2}{\pi} \int_{0}^{-\infty} \cos (2 \sigma \psi / a) e^{-n \psi^{2} / \sigma} d \psi \\
& =\sqrt{ }(6 / n \pi) e^{-6 \sigma^{2} / n a^{2}} d \sigma / a . . . .
\end{aligned}
$$

In comparing this with (5), we must observe that there $x$ denotes tie mean of the distances of which $\sigma$ is the sum, so t! at $\sigma=n x$, and thus the two results are in agreement.

If we denote the leading term in $\phi_{n}$ by $\Phi$, we cutain from (13) and (14)

$$
\phi_{n}=\Phi+6^{2} n h_{4} \frac{d^{2} \Phi}{d n^{2}}-6^{3} n h_{6} \frac{d^{3} \Phi}{d n^{3}}+\frac{1}{2} 6^{4} n^{2} h_{4}{ }^{2} \frac{d^{4} \Phi}{d n^{4}},
$$

by means of which the approximation in powers of $1 / n$ can be pursued. The terms written would suffice for a result correct to $1 / n^{2}$ inclusive, but we may content ourselves with the term which is of the order $1 / n$ in comparison with the leading term. We have

$$
\begin{aligned}
& \Phi=\sqrt{\left(\frac{6}{n \pi}\right) e^{-6 \sigma^{2} / n a^{2}},}
\end{aligned}
$$

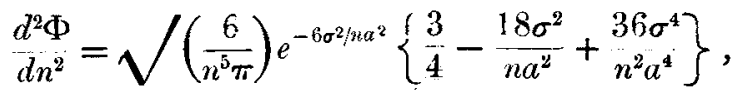

and accordingly

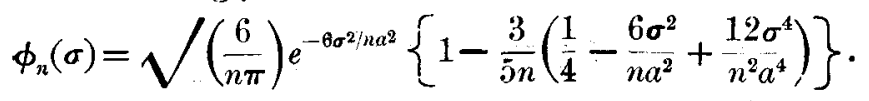

Here $\phi_{n}(\sigma) d \sigma / a$ expresses the probability that the sum of the distances, measured from the centre of the line, shall lie between $\sigma$ and $\sigma+d \sigma$.

In terms of the mean $(x)$ of the distances, we should have

$$
\sqrt{\left(\frac{6 n}{\pi}\right) e^{-6 n x^{2} / a^{2}}}\left\{1-\frac{3}{5 n}\left(\frac{1}{4}-\frac{6 n x^{2}}{a^{2}}+\frac{12 n^{2} x^{4}}{a^{4}}\right)\right\} d x / a
$$

as the probability that $x$ shall lie between $x$ and $x+d x$. It

* Phil. Mag. vol. xxxvii. p. 344.(1919), equations (65), (66), \&c. 
should be observed that in virtue of the exponential factor only moderate values of $n x^{2} / a^{2}$ need consideration.

Âs a check upon (19) we may verify that it becomes. unity when integrated with respect to $x$ between 0 and $\infty$. Starting from

$$
\sqrt{\left(\frac{u}{\pi}\right) \int_{0}^{\infty} e^{-u x^{2}} d x=1}
$$

and differentiating with respect to $u$, we get

$$
\frac{2 u^{32}}{\pi^{1 / 2}} \int_{0}^{\infty} x^{2} e^{-u x^{2}} d x=1
$$

and differentiating again

$$
\frac{4 u^{5 / 2}}{3 \pi^{1 / 2}} \int_{0}^{\infty} x^{4} e^{-u r^{2}} d x=1
$$

Using these integrals in (19) with $a=1, u=6 n$, the required verification follows.

The above verification suggests a remark which may have a somewhat wide application. In many cases we can foresee that a facility function will have a form such as $\mathrm{A} e^{-u x^{2}} d x$, and then, since

$$
\mathrm{A} \int_{0}^{\infty} e^{-u x^{2}} d x=1
$$

it follows that $A=2 \sqrt{ }(u / \pi)$. According to this law, the expectation of $x$ is zero, but the expectation of $x^{2}$ is finite. if we know this latter expectation, we may use the knowledge to determine $u$. For

$$
\text { expectation of } x^{2}=2 \vee(u / \pi) \int_{0}^{\infty} x^{2} e^{-u x^{2}} d x=1 / 2 u \text {. }
$$

We may take an example from the problem, just considered, of the position of the centre of gravity of points distributed along a line. If $x_{1}, x_{2}, \ldots x_{n}$ be the coordinates of these points reckoned from the middle and $x$ that of the centre of gravity,

$$
\text { Mean } x^{2}=\iiint \ldots \frac{d x_{1} d x_{2} \ldots d x_{n}}{a^{n}} \frac{\left(x_{1}+x_{2}+\ldots+x_{n}\right)^{2}}{n^{2}},
$$

the integrations being in each case from $-\frac{1}{2} \alpha$ to $+\frac{1}{2} a$. Taking first the integration with respect to $x_{n}$, we find that

$$
\text { Mean } x^{2}=\frac{a^{2}}{12 n^{2}}+\text { the corresponding expression with } x_{n},
$$


so that

$$
\text { Mean } x^{2}=a^{2} / 12 n \text {. }
$$

Accordingly $u=6 n / a^{2}$, as in (19).

A similar argument might be employed for the law of facility of various resultants $(r)$ of $n$ unit vibrations with phases entirely arbitrary, starting with $\mathrm{A} e^{-u r^{2}} r d r$, and assuming that the mean value of $r^{2}$ is $n$.

My principal aim in attacking the above problem was an introduction to the question of random vibrations when the phases of the unit components are distributed along a circular arc not constituting an entire circle. When the circle is complete the solution has already been given ${ }^{*}$, and the same solution obviously applies when the circular arc covers any number of complete revolutions. All phases of the resultant are then equally probable, and the only question relates to the probability of various amplitudes, or intensities. But if the arc over which the representative points are distributed is not a multiple of $2 \pi$, all values of the resultant phase are not equally probable and the question is in many respects more complicated.

There is an obvious relation between the question of the resultant of random vibrations and that of the position of the centre of gravity of the representative points of the components. For if $\theta$ denote the phase of a unit component, the intensity of the resultant is given by

$$
\mathrm{R}^{2}=(\Sigma \cos \theta)^{2}+(\Sigma \sin \theta)^{2} .
$$

If we suppose unit masses placed at angles $\theta$ round the circular are of radius unity, the rectangular coordinates of the centre of gravity are

$$
\bar{x}=(\Sigma \cos \theta) / n, \quad-=(\Sigma \sin \theta) / n ;
$$

and $r$, the distance of the centre of gravity from the centre of the circle, is related to $\mathrm{R}$ according to $r=\mathrm{R} / n$. And in like manner the phase of the resultant corresponds with the angular position of the centre of gravity.

The analogy suggests that a mechanical arrangement might be employed to effect vector addition. A disk, supported after the manner of a compass-card, would carry the loads, and the resulting deflexion from the horizontal would be determined by mirror reading. Perhaps there would be a difficulty in securing adequate delicacy.

* Phil. Mag. vol. x. p. 73 (1880); Scientific Papers, vol. i. p. 491. See also Phil. Mag. vol. xxxvii, p. 344 (1919). 
To return to the theoretical question, if we suppose the circular are to be very small, we see that the probability of varions phases of the resultant, within the narrow limits imposed, follows the laws determined for the centre of gravity of points distributed at random along a straight line. In this case the amplitude of the resultant is $n$ to a high degree of approximation, $n$ being the number of unit components.

But when the circul, $r$ arc $(\alpha)$ is so large that $\sin \alpha$ deviates appreciably from $\alpha$, the question is materially altered. We may, however, frame an argument on the lines followed in equations (1) and (2). Thus with a replacing $a$ and $\beta$ replacing $b$, we have for the resultant whose amplitude is $R$ and phase (reckoned from the middle) $\Theta$,

$$
\begin{array}{r}
\mathrm{R} \sin \Theta=\sin \beta \cdot\left(\xi_{1}-\xi_{-1}\right)+\sin 2 \beta \cdot\left(\xi_{2}-\xi_{-2}\right) \\
+\ldots+\sin s \beta \cdot\left(\xi_{s}-\xi_{-s}\right) \cdot . \cdot \cdot \cdot \\
\mathrm{R} \cos \Theta=\cos \beta \cdot\left(\xi_{1}+\xi_{-1}\right)+\ldots+\cos s \beta \cdot\left(\xi_{8}+\xi_{-s}\right)
\end{array}
$$

Here $\Theta$ is a smail angle, whose probability is under consideration, but $R$ is in general large and may then be reckoned as if the distribution were uniform. Thus

and

$$
\mathrm{R}=\frac{2 n}{\alpha} \int_{0}^{\frac{2}{2} \alpha} \cos \beta d \beta=(2 n / \alpha) \sin \frac{1}{2} \alpha . \quad .
$$

$\Theta=\frac{\alpha}{2 n \sin \frac{1}{2} \alpha}\left\{\sin \beta\left(\xi_{1}-\xi_{-1}\right)+\ldots+\sin s \beta\left(\xi_{s}-\xi_{-s}\right)\right\}$.

By the rules of the Theory of Errors, we have

$$
\underset{\operatorname{Mod}^{2} \xi}{\operatorname{Mod}^{2} \xi}=\frac{\alpha^{2}}{2 n^{2} \sin ^{2} \frac{1}{2} \alpha}\left\{\sin ^{2} \beta+\sin ^{2} 2 \beta+\ldots+\sin ^{2} s \beta\right\} \text {. }
$$

In (24) $\operatorname{Mod}^{2} \xi=2 n \beta / \alpha$, as before, and the series of $(\sin )^{2}$ may be replaced by

Thus

$$
\frac{1}{\beta} \int_{0}^{1 j a} \sin ^{2} \beta d \beta=\frac{\alpha-\sin \alpha}{4 \beta^{-a}} .
$$

$$
\operatorname{Mod}^{2} \theta=\frac{\alpha(\alpha-\sin \alpha)}{4 n \sin ^{2} \frac{1}{2} \alpha} .
$$

If $\alpha$ is small, this reduces to $\alpha^{2} / 6 n$, as in (4). If $\alpha=\pi$, that is if the distribution be over a semicircle, we get $\pi^{2} / 4 n$. If we make $\alpha=2 \pi$ in (25), the result is indeterminate, since although $\sin \frac{1}{2} \alpha=0, n$ is infinite. There is a like indeterminateness when $\alpha$ is any multiple of $2 \pi$, and this was to be expected. When the arc of distribution consists 
of entire revolutions, the phase of the resultant is arbitrary. But if the arc differs, even a little, from an integral number of revolutions, there is a definite phase favoured for the resultant, and $\operatorname{Mod}^{2} \Theta$ diminishes as $n$ increases.

The case where the arc consists of entire revolutions is exceptional also as regards the amplitude, or intensity, of the resultant. As we know, in that case no definite value is approached, however great $n$ may be, and the expectation of intensity is $n$. But if there be a fractional part of a revolution outstanding, the intensity does tend to a definite value, that namely which corresponds to a uniform distribution over the are, and this value is proportional to the square of $n$.

We may go further and calculate what exactly is the expectation of intensity. We have to evaluate

$$
\begin{aligned}
& \iiint \ldots \frac{d \theta}{\alpha} \frac{d \theta^{\prime}}{\alpha} \frac{d \theta^{\prime \prime}}{\alpha} \ldots\left[\left(\cos \theta+\cos \theta^{\prime}+\cos \theta^{\prime \prime}+\ldots\right)^{2}\right. \\
& \left.+\left(\sin \theta+\sin \theta^{\prime}+\sin \theta^{\prime \prime}+\ldots\right)^{2}\right] \\
& =\frac{1}{a^{\prime \prime}} \iiint \ldots d \theta d \theta^{\prime} d \theta^{\prime \prime} \ldots\left[n+2 \cos \left(\theta-\theta^{\prime}\right)\right. \\
& \left.\quad+2 \cos \left(\theta-\theta^{\prime \prime}\right)+\ldots+2 \cos \left(\theta^{\prime}-\theta^{\prime \prime}\right)+\ldots\right],
\end{aligned}
$$

the integration being in each case from $-\frac{1}{2} \alpha$ to $+\frac{1}{2} \alpha$. Taking first the integration with respect to $\theta$, we have

$$
\begin{aligned}
& \frac{1}{\alpha} \int_{-\frac{1}{2} \alpha}^{+\frac{1}{2} \alpha} d \theta\left[n+2 \cos \left(\theta-\theta^{\prime}\right)\right.+2 \cos \left(\theta-\theta^{\prime \prime}\right)+\ldots \\
&\left.+2 \cos \left(\theta^{\prime}-\theta^{\prime \prime}\right)+\ldots\right] \\
&=4 \alpha^{-1} \sin \frac{1}{2} \alpha\left\{\cos \theta^{\prime}+\cos \theta^{\prime \prime}+\ldots\right\}+n+2 \cos \left(\theta^{\prime}-\theta^{\prime \prime}\right)+\ldots .
\end{aligned}
$$

On continuing the integration the first part yields finally

$$
8(n-1) \alpha^{-2} \sin ^{2} \frac{1}{2} \alpha
$$

while the remaining parts give the original terms over again with omission of those containing $\theta$. Thus

Expectation of intensity

$$
\begin{aligned}
& =n+\gamma \alpha^{-2} \sin ^{2} \frac{1}{2} \alpha\{n-1+n-2+n-3+\ldots+1\} \\
& =n+4 n(n-1) \alpha^{-2} \sin ^{2} \frac{1}{2} \alpha . . . . . . . . .
\end{aligned}
$$

If $\alpha=0$, this becomes $n^{2}$, as was to be expected. If $\alpha=2 \pi$, or any multiple of $2 \pi$, the expectation is $n$, as we knew. In general, when $\alpha$ becomes great, so as to include many complete revolutions, the importance of the $n^{2}$ part decreasis. In (27) $n$ may have any integral value. 
In the case of $n=2$, we may go further and find the expression for the probability of a given amplitude $(r)$ taken always positive, and phase $(\theta)$. The amplitude of the components is unity, and the phases, measured from the centre of the arc, $\theta_{1}$ and $\theta_{2}$. The probability that these phases shall lie between $\theta_{1}$ and $\theta_{1}+d \theta_{1}, \theta_{2}$ and $\theta_{2}+d \theta_{2}$ is $\alpha^{-2} d \theta_{1} d \theta_{2}$. We have now to replace the two variables $\theta_{1}, \theta_{2}$ by $r, \theta$, where

or

$$
\begin{aligned}
r & =2 \cos \frac{1}{2}\left(\theta_{1}-\theta_{2}\right), \quad \theta=\frac{1}{2}\left(\theta_{1}+\theta_{2}\right), \\
\theta_{1} & =\theta \pm \cos ^{-1}\left(\frac{1}{2} r\right), \quad \theta_{2}=\theta \mp \cos ^{-1}\left(\frac{1}{2} r\right),
\end{aligned}
$$

making

$$
\frac{d \theta_{1}}{d \theta}=1, \quad \frac{d \theta_{2}}{d r}=\frac{ \pm 1}{\sqrt{\left(4-r^{2}\right)}}, \quad \frac{d \theta_{1}}{d r}=\frac{\mp 1}{\sqrt{\left(4-r^{2}\right)}}, \quad \frac{d \theta_{2}}{d \theta}=1 .
$$

Accordingly

$$
\frac{d \theta_{1} d \theta_{2}}{a^{2}}=\frac{ \pm 2 d \theta d r}{\alpha^{2} \sqrt{\left(4-r^{2}\right)}}, \quad . \quad .
$$

The interchange of $\theta_{1}$ and $\theta_{2}^{\bullet}$ makes no difference to $r$ and $\theta$, so that we may take

$$
\frac{4 d \theta d r}{\alpha^{2} \frac{\sqrt{\left(4-r^{2}\right)}}{2}}, \ldots . . . .
$$

as the chance that the amplitude of the resultant shall lie between $r$ and $r+d r$ and the phase between $\theta$ and $\theta+d \theta$. In (29) $\alpha$ is supposed not to exceed $2 \pi$.

As a check, we may revert to the case where $\alpha=2 \pi$. The limits for $\theta$ are then independent of the value of $r$, and are taken to be $-\pi$ and $+\pi$. And

$$
\frac{4 d r}{\sqrt{ }\left(4-\overline{\left.r^{2}\right)}\right.} \int_{-\pi}^{+\pi} \frac{d \theta}{\alpha^{2}}=\frac{2}{\pi} \frac{d r}{\sqrt{\left(4-r^{2}\right)}}=\frac{2 \pi r d r}{\pi^{2} r \sqrt{\left(4-r^{2}\right)}}
$$

represents the chance that $r$ shall lie between $r$ and $r+d r$ independently of what $\theta$ may be, in agreement with Pearson's expression *. Integrating a gain with respect to $r$, we find

$$
\int_{0}^{2} \frac{2 d r}{\pi \sqrt{\left(4-r^{2}\right)}}=1
$$

as should be, all cases being now covered.

In the general case the limits for $r$ and $\theta$ are interdependent. The possible range for $\theta$ is from $-\frac{1}{2} \alpha$ to $+\frac{1}{2} \alpha$ $(\alpha<\pi)$, but we require the range when $r$ is prescribed. In virtue of the symmetry it suffices to consider a positive $\theta$,

* Compare Phil. Mag. vol, xxxvii. p. 328 (1919), equation (21). 
and we begin by supposing a less than $\pi$, so that the extreme values of $r$ are $2 \cos \frac{1}{2} \alpha$ and 2 . We proceed to consider the relations by which the limiting values of $r$ and $\theta$ are connected.

For a given (positive) $\theta$ less than $\frac{1}{2} \alpha$ the upper limit of $r$ is 2 and the lower limit is $2 \cos \left(\frac{1}{2} \alpha-\theta\right)$. When $\theta>\frac{1}{2} \alpha$, there are no corresponding values of $r$. In fig. 2 , where $\alpha$ is taken to be $\frac{1}{2} \pi$, the shaded area gives the possible values of $r$ corresponding to any $\theta$, or conversely the values of $\theta$ corresponding to a prescribed $r$.

In order to find the chances of a given $\theta$, we integrate with respect to $r$ in (29). We find

$$
\frac{4 d \theta}{\alpha^{2}} \int_{2 \cos \left(\frac{1}{2} \alpha-\theta\right)}^{2} \frac{d r}{\sqrt{ }\left(4-r^{2}\right)}=\frac{4 d \theta}{\alpha^{2}}\left(\frac{1}{2} \alpha-\theta\right),
$$

as the chance that $\theta$, if positive, lies between $\theta$ and $\theta+d \theta$. If we integrate (31) again with respect to $\theta$ between 0 and $\frac{1}{2} \alpha$, we get $\frac{1}{2}$, the correct value, as there is an equal chance of $\theta$ being negative.

Again, in order to find the chance of a prescribed $r$, when $\theta$ is free to vary, we have to integrate (29) first with respect to $\theta$. Referring to fig. 2 , we see that when $r<2 \cos \left(\frac{1}{2} \alpha\right)$, there are no corresponding values of $\theta$, and that when $r$ lies between $2 \cos \left(\frac{1}{2} \alpha\right)$ and 2 , the limits for $\theta$ are 0 and $\frac{1}{2} \alpha-\cos ^{-1}\left(\frac{1}{2} r\right)$. In the first case there is no possibility of $r$ lying between $r$ and $r+d r$; in the second case the probability is

$$
\frac{4 d r}{\alpha^{2} \sqrt{\left(4-r^{2}\right)}}\left\{\frac{1}{2} \alpha-\cos ^{-1}\left(\frac{1}{2} r\right)\right\}, \quad . \quad \text {. . }
$$

which must be doubled when we admit, as we must, negative values of $\theta$. If we integrate (32) as it stands, again with respect to $r$, we find the correct value, since

$$
\int_{2 \cos \frac{1}{2} \alpha}^{2} \frac{4 d r}{\alpha^{2} \sqrt{ }\left(4-r^{2}\right)}\left\{\frac{1}{2} \alpha-\cos ^{-1}\left(\frac{1}{2} r\right)\right\}=\frac{1}{2} .
$$

We may regard (31) and (32) as the solution of the problem in the case where $\alpha<\pi$.

When $\alpha>\pi, \theta$ may lie outside the limits $\pm \frac{1}{2} \alpha$ applicable to $\theta_{1}$ and $\theta_{2}$, and the question becomes more complicated. It appears that we must distinguish two cases under this head, (i.) where $\pi<a<3 \pi / 2$, and (ii.) where $3 \pi / 2<\alpha<2 \pi$.

First for $\pi<\alpha<3 \pi / 2$, fig. 4, where $\alpha$ is supposed to be $5 \pi / 4$. 

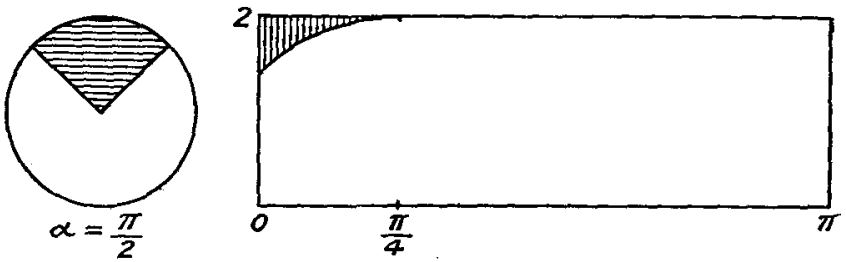

Fig. 2 .
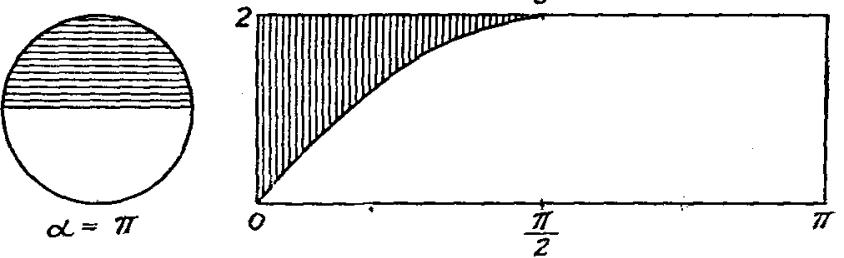

Fig. 3.
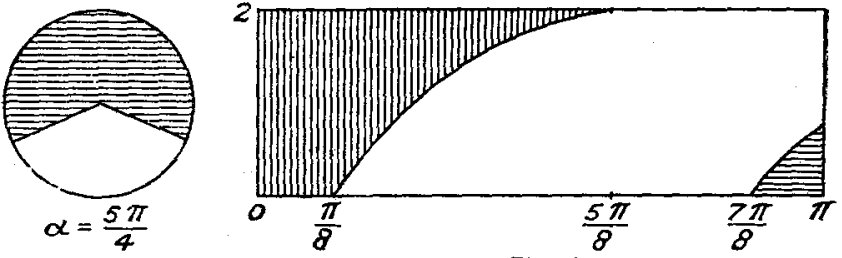

Fig. 4 .
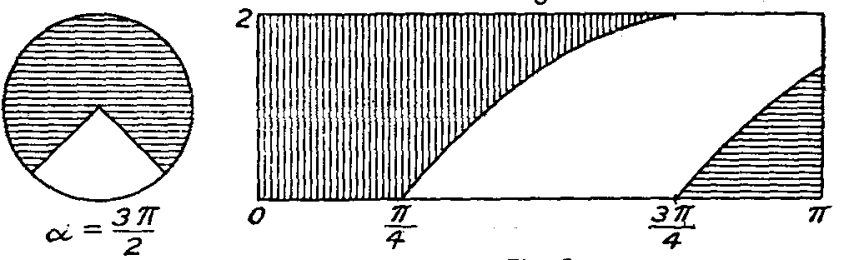

Fig. 5
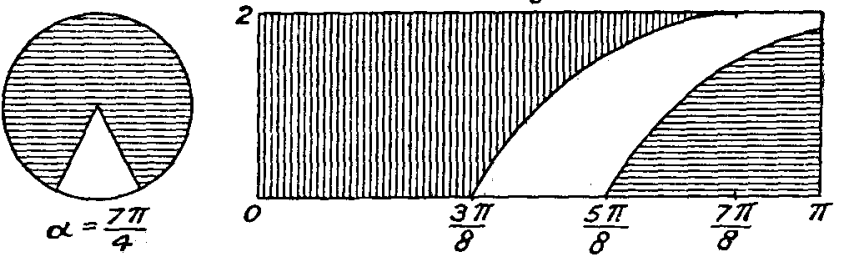

Fig. 6.
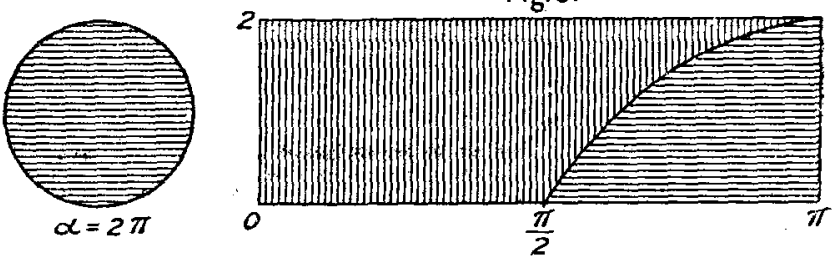

Fig. 7. 
From $\theta=0$ to $\theta=\frac{1}{2}(\alpha-\pi), r$ ranges from 0 to 2 . From $\theta=\frac{1}{2}(\alpha-\pi)$ to $\theta=\frac{1}{2} \alpha, r$ ranges from $2 \cos \left(\frac{1}{2} \alpha-\theta\right)$ to 2 . At $\theta=\frac{1}{2} \alpha$ the lower and upper limits coincide. From $\theta=\frac{1}{2} \alpha$ to $\theta=3 \pi / 2-\frac{1}{2} \alpha$, there are no corresponding values of $r$. At the latter limit a zero value of $r$ enters, and from $\theta=3 \pi / 2-\frac{1}{2} \alpha$ to $\theta=\pi, r$ ranges from 0 to $2 \cos \left(2 \pi-\frac{1}{2} \alpha-\theta\right)$.

The whole range from $\theta=0$ to $\theta=\pi$ thus divides itself into four parts. In the first part from $\theta=0$ to $\theta=\frac{1}{2}(\alpha-\pi)$, we get as the chance of $\theta$ from (29)

$$
\frac{4 d \theta}{\alpha^{2}} \int_{0}^{2} \frac{d r}{\sqrt{\left(4-r^{2}\right)}}=\frac{2 \pi d \theta}{\alpha^{2}}, . . .
$$

In the second part from $\theta=\frac{1}{2}(\alpha-\pi)$ to $\theta=\frac{1}{2} \alpha$, the chance is

$$
\frac{4 d \theta}{\alpha^{2}} \int_{2 \cos \left(\frac{2}{2} \alpha-\theta\right)}^{2} \frac{d r}{\sqrt{\left(4-r^{2}\right)}}=\frac{4 d \theta}{\alpha^{2}}\left(\frac{1}{2} \alpha-\theta\right) .
$$

For the third part, from $\theta=\frac{1}{2} \alpha$ to $\theta=3 \pi / 2-\frac{1}{2} \alpha$, there is no possibility.

For the fourth part, from $\theta=3 \pi / 2-\frac{1}{2} \alpha$ to $\theta=\pi$, the chance for $\theta$ is

$$
\frac{4 d \theta}{\alpha^{2}} \int_{0}^{2 \cos \left(2 \pi-\frac{1}{2} \alpha-\theta\right)} \frac{d r^{2}}{\sqrt{\left(4-r^{2}\right)}}=\frac{4 d A}{\alpha^{2}}\left(\frac{1}{2} \alpha+\theta-\frac{3 \pi}{2}\right) .
$$

If we integrate (33), (34), and (35) over the (positive) ranges to which they apply and add the results, we get the correct value, viz. $\frac{1}{2}$. This part of the question might be treated more simply without introducing $r$ at all.

We have next to consider what in this case, viz. $\pi<x<3 \pi / 2$, are the probabilities of various $r$ 's when $\theta$ is allowed to vary. When $r$ is less than its value at $\theta=\pi$, viz. $2 \cos \left(\pi-\frac{i}{2} \alpha\right)$, the corresponding range for $\theta$ is male up of two parts, the first from $\theta=0$ to $\theta=\frac{1}{2} \alpha-\cos ^{-1}\left(\frac{1}{2} r\right)$, and the second from $\theta=2 \pi-\frac{1}{2} \alpha-\cos ^{-1}\left(\frac{1}{2} r\right)$ to $\theta=\pi$, so that the whole range of $\theta$ is

$$
\frac{1}{2} \alpha-\cos ^{-1}\left(\frac{1}{2} r\right)+\pi-\left\{2 \pi-\frac{1}{2} \alpha-\cos ^{-1}\left(\frac{1}{2} r\right)\right\}=\alpha-\pi .
$$

Thus from $r=0$ to $r=2 \cos \left(\pi-\frac{1}{2} \alpha\right)$ the chance of $r$ lying between $r$ and $r+d r$ is

$$
\frac{4 d r(\alpha-\pi)}{\alpha^{2} \vee\left(4-r^{2}\right)} \text {. . . . . . . }
$$

When $r$ lies between $2 \cos \left(\pi-\frac{1}{2} \alpha\right)$ and 2 , the second part disappears and we have only the one range of $\theta$, equal to Phil. Mag. S. 6. Vol. 37. No. 221. May 1919. $2 \mathrm{~N}$ 
514 On the Resultant of a Number of Cnit Vibrations. $\frac{1}{2} \alpha-\cos ^{-1}\left(\frac{1}{2} r\right)$, so that the chance of $r$ lying between $r$ and $r+d r$ is

$$
\frac{4 d r\left\{\frac{1}{2} \alpha-\cos ^{-1}\left(\frac{1}{2} r\right)\right\}}{\alpha^{2} \sqrt{2}\left(4-r^{2}\right)} . . . . .
$$

Expressions (36) and (37), obtained on the supposition that $\theta$ is positive, are to be doubled when we allow for the equally admissible negative values of $\theta$.

When (36), (37), as they stand, are integrated over the ranges of $r$ to which they apply and added, the sum is $\frac{1}{2}$, as it should be under the suppositions made.

It still remains to consider the case where $3 \pi / 2<\alpha<2 \pi$. From $\theta=0$ to $\theta=\frac{1}{2}(\alpha-\pi), r$ (as before) ranges from 0 to 2 . From $\theta=\frac{1}{2}(\alpha-\pi)$ to $\theta=\frac{1}{2}(3 \pi-\alpha), r$ ranges from $2 \cos \left(\frac{1}{2} \alpha-\theta\right)$ to 2. At this point (fig. 6) a second range enters for $r$. From $\theta=\frac{1}{2}(3 \pi-\alpha)$ to $\theta=\frac{1}{2} \alpha$, the first range is, as before, from $2 \cos \left({ }_{2}^{1} \alpha-\theta\right)$ to 2 , and the second range is from 0 to $2 \cos \left(2 \pi-\frac{1}{2} \alpha-\theta\right)$. Lastly, from $\theta=\frac{1}{2} \alpha$ to $\theta=\pi$, the first range of $r$ disappears, while the second continues to be from 0 to $2 \cos \left(2 \pi-\frac{1}{2} \alpha-\theta\right)$.

The probabilities of various $\theta$ 's being positive and lying within specified ranges can be obtained as before. For the range from $\theta=0$ to $\theta=1(\alpha-\pi)$ we get the expression (33), and from $\theta=\frac{1}{2}(\alpha-\pi)$ to $\theta=\frac{1}{2}(3 \pi-\alpha)$ we get (34). For the third range from $\theta=\frac{1}{2}(3 \pi-\alpha)$ to $\theta=\frac{1}{2} \alpha$, we get

$$
\begin{aligned}
\frac{4 d \theta}{u^{2}}\left[\int_{2 \cos \left(\frac{1}{2} \alpha-\theta\right)}^{2}\right. & \left.+\int_{0}^{2 \cos \left(2 \pi-\frac{1}{2} \alpha-\theta\right)}\right] \frac{d r}{\sqrt{\left(4-r^{2}\right)}} \\
& =\frac{4 d \theta}{\alpha^{2}}\left(\alpha-\frac{3 \pi}{2}\right) ; . . . . .
\end{aligned}
$$

and from $\theta=\frac{1}{2} \alpha$ to $\theta=\pi$,

$$
\frac{4 d \theta}{\alpha^{2}} \int_{0}^{2 \cos \left(2 \pi-\frac{1}{2} \alpha-\theta\right)} \frac{d r}{\sqrt{\left(4-r^{2}\right)}}=\frac{4 d \theta}{\alpha^{2}}\left(\frac{1}{2} \alpha+\theta-\frac{3 \pi}{2}\right) .
$$

If the integrations with respect to $\theta$ are effected over the appropriate ranges and the results added, we get $\frac{1}{2}$, as was to be expected.

Finally, for the probabilities of various $r$ 's when $\theta$ is left open, we get for $r$ between 0 and $2 \cos \left(\pi-\frac{1}{2} \alpha\right)$ two ranges for $\theta$, viz. from 0 to $\frac{1}{2} \alpha-\cos ^{-1}\left({ }_{2}^{3} r\right)$, and again, from $\theta=2 \pi-\frac{1}{2} \alpha-\cos ^{-1}\left(\frac{1}{2} r\right)$ to $\theta=\pi$, making altogether $(\alpha-\pi)$. Thus for these values of $r$ the probability is that expressed in (36).

When $r$ lies between $2 \cos (\pi-1)$ and 2 , we recover in like manner (37). And as before we may verify the results 
Whirling of an Excentrically-loaded Overhung Shaft. 515

by showing that when the second integrations are carried out over the appropriate ranges and the integrals added, we recover $\frac{1}{2}$.

It may be remarked that the latter results may be applied to the complete circle by making $\alpha=2 \pi$ (fig. 7). The second range for $r$ then disappears, and for the whole range now extending for all values of $\theta$ from $r=0$ to $r=2$ we get

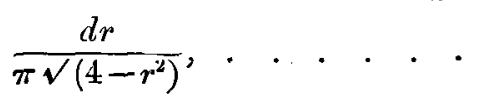

which needs to be doubled in order to take account of negative values of $\theta$.

'This completes the investigation for an arbitrary $\alpha$ (less than $2 \pi$ ), when $n=2$. Since even for the complete circle $(\alpha=2 \pi)$ the case $n=3$ leads to elliptic integrals, there is no encouragement to try an extension to other values of $\alpha$.

Terling Place, Witham.

March 31, 1919.

XLVII. The Whirling of an Excentrically-loaded Overhung Shaft. By S. LeEs, M.A., College of Technology, Man.chester *

$\S 1 . T$ THE general problems arising in connexion with the whirling of shafts have been treated at some length by several investigators, prominent amongst whom may be mentioned Greenhill †, Dunkerley $\ddagger$, and Chree $\$$. 'The latter, in particul' $\mathbf{r}$, has given a very elaborate treatment of the subject on mathematical lines, but does not give any attention to the practial problem of the whirling of shafts with excentric loads.

$\$ 2$. In what follows, an attempt is made to deduce the dynamical equations giving the motion of such an overhung, excentrically-loaded shaft. The writer has taken into account the influence of the rotational inertia of the whirling load, but has made what seems a reasonable assumption, namely, that the whirling load can be taken as a perfectly true Hywheel, mounted slightly excentrically. This simplifies the mathematical discussion somewhat. It is also further assumed that the weight of the shaft can be neglected.

* Communicated by the Author.

$\div$ Greenhill, Proc. Inst. Mech. Eng. 1883.

+ Dunlierley, Phil. Trans. Roy. Suc. A, 1894 .

$\$$ Chree, Phil. Mag. May 1904. 